\title{
Porcelain laminate veneer - a conservative treatment in restoration of anterior teeth defect
}

\author{
F Rashid
}

\begin{abstract}
In this study, $100 \%$ of the patients were satisfied aesthetically after 6 months and 12 months. $100 \%$ patients presented without the complaint of sensitivity after 6 and 12 months. $100 \%$ of the patients presented with healthy periodontium after 6 and 12 months. De bonding and porcelain fracture were found in $10 \%$ in both the cases after 12 months. No complaint against aesthetic and sensitivity was recorded after 12 months.
\end{abstract}

Keywords: Porcelain laminate veneer, aesthetics, anterior tooth defect, conventional crown.

\section{Introduction}

A defect in anterior teeth requires special attention to aesthetic. Currently porcelain laminate veneer is the most durable aesthetic material to correct those that are discolored, miss-happened or damaged. Porcelain laminate veneer is probably the most aesthetic means of creating a more pleasing and beautiful smile. ${ }^{1}$ In many cases veneers are applied instead of mostly and invasive crowns. $^{2}$

Veneers are a permanent process and should therefore only be considered by individuals that have all of their permanent teeth. Porcelain laminate veneers are extremely thin pieces of Ceramic material that are permanently attached to the front of a patient's teeth using a Composite resin as bonding cement ${ }^{3}$, in order to enhance the union between the tooth and Porcelain laminate veneer, the development of acid - etching of enamel to improve the retention of resin first described by Buonocore ${ }^{4}$, which attaching veneers to the teeth by less destructive means. The inner surface of the porcelain laminate veneer is treated with hydrofluoric acid etching till its frosty white and increasing the surface area with retentive irregularities for a mechanical bond to composite.

\footnotetext{
Address of Correspondence:

Dr. Fouzia Rashid, MS, Associate Professor \& Head of the Department of Prosthodontics, Pioneer Dental College and Hospital, Dhaka, Bangladesh.

E-mail: diprorahman85@gmail.com
}

Aesthetic veneer should always be considered as a conservative alternative to cemented crowns. In many practices, they have largely replaced metal ceramic crowns. ${ }^{5}$ Un-questionably; one of the disadvantages of conventional crown is the destruction of tooth structure. An advantage of this approach is the saving of tooth structure, laboratory time and materials, thus giving a possible cost advantage.

\section{Materials and Methods}

This prospective study was conducted in the Department of Prosthodontics, Faculty of Dentistry, Banghabandhu Sheikh Mujib Medical University (BSMMU), Shahbag, Dhaka from January 2004 to December 2005. 30 patients were selected who attended in this department for treatment of discolored anterior teeth, abrasion, erosion, minor mal-alignment and anterior teeth diastema as the subjects of this study. Each patient was evaluated by a thorough medical and dental history as well as clinical and radiographic examination.

According to patient's concern he or she was selected for the treatment and tooth preparation was done. A minimum $0.5 \mathrm{~mm}$ labial tooth surface reduction should be done which follow the anatomic contours of tooth. Interproximal preparation reached the facial aspect of the contact area to ensure that the margin between veneer and tooth surface was not visible. Enamel was preserved in the interproximal contact area. The incisal edge was extended lingually only if a diastema or minor mal- alignment of anterior tooth was recorded. An accurate impression of the prepared teeth was taken with elastomeric impression and master cast was poured. A refractory was made by the duplication of master cast by type 4 dental stone. 
Die spacer was applied to the labial surfaces and $2 \mathrm{~mm}$ was short of the finish line. Porcelain was build-up incrementally and contoured with the reference to the study model which was taken before tooth preparation. Porcelain powder build-up was done in layers for firing (Firing was done above $900^{\circ} \mathrm{C}$ ).

After completion of build-up, firing and contouring, the refractory dies were separated individually by a thin diamond disc. The veneer was then glazed. The final prosthesis was cemented on etched tooth surface and restoration was seated with a composite resin luting agent. Having cemented patients were advised to attend after 6 and 12 months for the evaluation of both the prosthesis and associated oral tissues. Data collection was done according to specific parameters like esthetics, sensitivity development, periodontal disease, marginal integrity, porcelain fracture and de-bonding. The findings were recorded in a pre-designed data collection sheet.

\section{Results}

The table shows the variables and results recorded in this study after 6 months and 12 months.

\begin{tabular}{|c|c|c|c|c|}
\hline \multirow{2}{*}{ Variables } & \multicolumn{2}{|c|}{ After 6 months } & \multicolumn{2}{c|}{ After 12 months } \\
\cline { 2 - 5 } & Affected \% $(\mathrm{No})$ & Not affected \% $(\mathrm{No})$ & Affected \% (No) & Not affected \%(No) \\
\hline Aesthetics & $00(00)$ & $100(30)$ & $00(00)$ & $100(30)$ \\
\hline Sensitivity & $00(00)$ & $100(30)$ & $00(00)$ & $100(30)$ \\
\hline Peridodontal diseases & $00(00)$ & $100(30)$ & $00(00)$ & $100(30)$ \\
\hline Marginal integity & $00(00)$ & $100(30)$ & $10(03)$ & $90(27)$ \\
\hline Porcelinin fracture & $6.7(02)$ & $93.3(28)$ & $10(03)$ & $90(27)$ \\
\hline De-bonding & $00(00)$ & $90(30)$ & $10(03)$ & $90(27)$ \\
\hline
\end{tabular}

In this study total 30 patients were treated with porcelain laminate veneer to find out any complications in one year. After 6 months only $6.7 \%$ patients of porcelain laminate veneer were affected by porcelain fracture. No patient complained about their aesthetic, sensitivity, periodontal disease after 6 and 12 months.

After 12 months of cementation there was no alteration of marginal integrity in case of $90 \%$ of the patients and $10 \%$ patients had alteration of marginal integrity. Porcelain fracture and debonding occured in $10 \%$ cases after 12 months. No patient complained about the aesthetic as there was no mis-match in color, shade and translucence between restoration and adjacent teeth.

\section{Discussion}

Majority of the patients in this study was female, a similar finding was also reported in other clinical evaluation on porcelain laminate veneers. This suggests that, in general, females might be more interested in their appearance that improves esthetics. ${ }^{6} \mathrm{~A}$ research organization has stated that Porcelain veneers will become an "accepted procedure" that promises "the highest esthetics potential to date for restoration of anterior tooth defect."

$100 \%$ of the patients presented without the complain of sensitivity after 12 months which is very advantageous point for porcelain laminate veneer. There was alteration of marginal integrity in case of $10 \%$ of the patients. Herbert Dumfahrt et al showed in a study that marginal integrity was acceptable in $99 \%$ cases among 191 patients and excellent in $63 \%$ case. $^{8}$ After a year of cementation $10 \%$ of the patients treated with porcelain laminate veneer presented with debonded prosthesis and other $90 \%$ were not affected. Swift et al reported that shear bond strength of composite luted to silanded porcelain laminate veneers would not be significantly affected by saliva or latex glove contamination. ${ }^{9}$ Other studies show that saliva contamination of either etched enamel or porcelain will reduced its bond strength to composite significantly. ${ }^{10}$

\section{Conclusion}

It can be concluded that the Porcelain Laminate veneer is an advantageous restoration for the improvement of aesthetic in anterior teeth which has minor surface defect, abrasion, erosion and discoloration as it is more conservative.

\section{References}

1. Stuart A.Greene. http://www.qualitydentistry.com/ dental/restorative/veneers $2 . h t m l$

2. Dr Khosla. http://members.rediff.com/ drkhosla/ veneers.html

3. Dr khosla. http://members.rediff.com / drkhosla / veneers.html

4.Buonocore MG.A simple method of increasing the adhesion of acrylic filling materials to enamel surfaces. Journal of Dental research 1995;34:849-53. 
5. S.F.Rosenstiel, Martin F, Junhei Fuzimoto,: Tooth preparation for all ceramic restoration, Contemporary fixed Prosthodontics, 3rd edition,Indian print: Mosby,2002:267.

6. Touati B, Bonded ceramic restorations-achieving predictability. Practical Periodontics and Aesthetic Dentistry1995;7:33-37.

7. Herbert Dumfahrt, Porcelain Laminate Veneers. A retrospective Evaluation After 1 to 10years of Service: Part 1-Clinical Procedure. The international journal of Prosthodontics 1995;12:511.
8. Herbert Dumfahrt, Porcelain Laminate Veneers. A retrospective Evaluation After 1 to 10years of service: Part 2-Clinical Results. The international journal of Prosthodontics 2000;13:14.

9. Swft et al. The Effect of Bond Strength of Composite Luting to Porcelain Laminate Veneer. Dental Materials 1994;10:278-81.

10. Herbart Dumfahrt, Porcelain Laminate Veneers. A retrospective Evaluation After 1 to 10years of service: Part 2-Clinical results. The international journal of Prosthodontics 2000;13:14. 